University of Nebraska - Lincoln

DigitalCommons@University of Nebraska - Lincoln

$4-2000$

\title{
Juvenile Hormone Titer and Morph-Specific Reproduction in the Wing-Polymorphic Cricket, Gryllus firmus
}

\author{
Gretchen Cisper \\ University of Nebraska - Lincoln \\ Anthony J. Zera \\ University of Nebraska - Lincoln, azera1@unl.edu \\ David W. Borst \\ Illinois State University, Normal, IL
}

Cisper, Gretchen; Zera, Anthony J.; and Borst, David W., "Juvenile Hormone Titer and Morph-Specific Reproduction in the Wing-Polymorphic Cricket, Gryllus firmus" (2000). Anthony Zera Publications. 37. https://digitalcommons.unl.edu/bioscizera/37

This Article is brought to you for free and open access by the Papers in the Biological Sciences at DigitalCommons@University of Nebraska - Lincoln. It has been accepted for inclusion in Anthony Zera Publications by an authorized administrator of DigitalCommons@University of Nebraska - Lincoln. 
Published in Journal of Insect Physiology 46:4 (April 2000), pp. 585-596; doi 10.1016/S0022-1910(99)00144-4

Copyright @ 2000 Elsevier Science Ltd. Used by permission. http:// www.sciencedirect.com/science/journal/00221910

Submitted January 19, 1999; accepted June 2, 1999; published online February 14, 2000.

\title{
Juvenile hormone titer and morph-specific reproduction in the wing-polymorphic cricket, Gryllus firmus
}

\author{
Gretchen Cisper, ${ }^{1}$ Anthony J. Zera, ${ }^{1}$ and David W. Borst ${ }^{2}$ \\ 1 School of Biological Sciences, University of Nebraska-Lincoln, Lincoln, NE 68588, USA \\ 2 Department of Biological Sciences, Illinois State University, Normal, IL 61761, USA \\ Corresponding author - A. J. Zera, azera@unl.edu
}

\begin{abstract}
Juvenile hormone titers and reproductive characteristics were measured in adult wing and flight-muscle morphs of the wing-polymorphic cricket, Gryllus firmus, during the first week of adulthood. This species has three morphs: one flight capable morph with fully-developed wings and fully-developed flight muscles [LW(F)], one flightless morph with fullydeveloped wings and histolyzed (non-functional) flight muscles [LW(H)], and another flightless morph with underdeveloped (short) wings and underdeveloped flight muscles (SW). Both flightless morphs [LW(H) and SW] had larger ovaries which contained a greater number of postvitellogenic eggs compared with the flight capable [LW(F)] morph. The juvenile hormone titer was significantly higher in SW compared with LW(F) females on days 3-7 of adulthood. On these days, the JH titer also was significantly higher in the other flightless morph, LW(H), compared with flight-capable $[\mathrm{LW}(\mathrm{F})]$ females as determined by one statistical test, but did not differ significantly by another test. The JH titer was positively correlated with ovarian mass or terminal oocyte length, but not with the number of post-vitellogenic eggs. This study is the first direct comparison of juvenile hormone titers in adult wing morphs of a wing-polymorphic insect. Results indicate that an elevated juvenile hormone titer may be at least partly responsible for one of the most distinctive features of wing-polymorphic species, the increased early fecundity of flightless females.
\end{abstract}

Keywords: juvenile hormone, JH, JH III, wing polymorphism, reproduction, Gryllus, crickets

\section{Introduction}

Wing polymorphism occurs widely in the Insecta (Harrison, 1980; Roff, 1986; Dingle, 1996; Zera and Denno, 1997). The polymorphism involves discontinuous variation in a wide variety of traits involved in dispersal and reproduction. Polymorphic species contain a flightless morph with underdeveloped wings and flight muscles. An alternate morph has fully developed wings, fully developed flight muscles shortly after the adult molt, and is capable of flight. Importantly, flight capability trades off with ovarian growth: flightless adults begin egg development earlier and typically produce more eggs, especially during the early stages of reproduction, than their flight-capable counterpart (Harrison, 1980; Roff, 1986; Zera and Denno, 1997). Wing polymorphism has been used extensively by evolutionary biolo- gists as a model to study the evolution of dispersal, development, and life histories and by insect physiologists to study the endocrine regulation of alternate developmental pathways within species (Hardie and Lees, 1985; Nijhout, 1994; Dingle, 1996; Zera and Denno, 1997).

Despite decades of study, the endocrine regulation of wing-polymorphism remains a largely unresolved problem in insect endocrinology (Hardie and Lees, 1985; Zera and Denno, 1997). At issue are the endocrine mechanisms that regulate the differential development and reproduction of alternate morphs within a species. The central focus of this topic has been juvenile hormone $(\mathrm{JH})$, a key regulator of insect development and reproduction (Nijhout, 1994; Wyatt and Davey, 1996). During the standard molting cycle, $\mathrm{JH}$, above some threshold, causes retention of juvenile features by blocking the metamorphic effect of ecdysone. During the last sta- 
dium, the JH titer drops to a very low level and ecdysone-mediated metamorphosis ensues. In most insect orders, JH takes on a different function in adults, where it positively regulates many aspects of reproduction such as the synthesis of vitellogenin and the uptake of this molecule into developing oocytes (Nijhout, 1994; Wyatt and Davey, 1996).

Decades ago, Wigglesworth (1961) and Southwood (1961) proposed that the short-winged (or wingless) morph of wing polymorphic species is produced by an elevated $\mathrm{JH}$ titer during some critical stage in development. This elevated titer purportedly blocks the full development of wings, flight muscles and associated structures thus giving rise to a short-winged or wingless morph. Since JH positively affects reproduction, an elevated $\mathrm{JH}$ titer in the short-winged adult would also account for its earlier and greater egg production (Roff, 1986; Zera and Denno, 1997).

Numerous studies have attempted to indirectly test this hypothesis. Variation in endogenous JH titers between morphs has been inferred from the effect of grosslevel experimental manipulations of the JH titer (Hardie and Lees, 1985; Mittler, 1991; Zera and Denno, 1997, and references therein). For a variety of reasons, results from these indirect studies have been equivocal (Zera and Denno, 1997; see Section 4). Clearly, more definitive evidence on the role of $\mathrm{JH}$ in regulating aspects of wing polymorphism requires direct quantification and comparison of $\mathrm{JH}$ titers between morphs during juvenile and adult stages. Thus far, no such direct comparisons of JH titers have been performed for adult morphs and only one comparison has been performed for juvenile morphs (Zera et al., 1989; Zera and Denno, 1997).

The present investigation represents part of a comprehensive study of the hormonal, energetic and biochemical bases of wing polymorphism in crickets of the genus Gryllus. One of our major goals is to identify the endocrine mechanisms responsible for the elevated egg production of the flightless morph relative to the flightcapable morph. Egg production is especially enhanced during early adulthood in flightless females of wingpolymorphic species (Roff, 1986; Zera and Denno, 1997). This elevated early fecundity typically is the major contributor to the overall greater fecundity of flightless females (Roff, 1986, 1986; Zera, 1984; Denno et al., 1989; Zera and Rankin, 1989; Zera and Denno, 1997). Hence, in the present study we focused on the endocrine regulation of the first stages of reproduction. Specifically, we compared juvenile hormone titers between long-winged and short-winged females of Gryllus firmus during the first week of adulthood when these morphs differ dramatically in ovarian growth. JH titers were measured using a well established radioimmunoassay (Hunnicutt et al., 1989) that is sensitive enough to quantify hemolymph titers in single individuals.
Importantly, in the present study, we not only quantified endocrine and reproductive characteristics of long-winged and short-winged adults, but also characterized these traits separately in the two functionally-differentiated types of long-winged individuals. In many wing polymorphic species, including G. firmus, long-winged adults are a composite group consisting of individuals that retain fully-developed flight muscles and those that histolyze their flight muscles when ovarian growth commences (Zera and Denno, 1997). These two types of long-winged morphs typically have not been distinguished in previous studies of wing polymorphism (e.g. Roff, 1984; Zera and Rankin, 1989). However, a recent study has shown that longwinged individuals with histolyzed flight muscles are much more similar to short-winged individuals in a variety of key physiological and reproductive characteristics than they are to long-winged individuals that retain their flight muscles (Zera et al., 1997; see Section 4). Consequently, in the present study, endocrine and reproductive features were measured separately for each of the two types of long-winged morphs, in addition to the short-winged morph.

\section{Materials and methods}

\subsection{Chemicals}

All chemicals and solvents used in the present study were at least reagent or HPLC grade and were purchased from Sigma Chemical Company, Mallinckrodt, or Fisher Scientific. Silica gel Prep-Sep extraction columns were purchased from Fisher Scientific. Racemic unlabeled JH III and labeled $\left[10^{-3} \mathrm{H}\right] \mathrm{JH}$ III $(19.5 \mathrm{Ci} /$ $\mathrm{mmol} ; 721.5 \mathrm{GBq} / \mathrm{mmol}$ ) were purchased from Sigma Chemical Co. and New England Nuclear, respectively.

\subsection{Insects, morph designations and rearing conditions}

Gryllus firmus, the sand cricket, occurs in the southeastern United States as a long-winged (LW) morph, some of which are capable of flight, or as a short-winged (SW) form that is obligatory flightless (Veazy et al., 1976). All SW females have white, non-functional flight muscles which never fully develop. All LW females have fully-developed, functional (pink) flight muscles at or shortly after the adult molt and are denoted as LW(F). During adulthood, some LW(F) individuals histolyze their flight muscles thus becoming flightless [denoted as $\operatorname{LW}(\mathrm{H})$; for review see Zera et al., 1997). The G. firmus used in the present study were derived from a colony founded from 30 gravid females collected in Gainesville, Florida during the summer of 1995. This base population was used to initiate a selection exper- 
iment on wing morph, the details of which will be reported elsewhere. Results presented here are for two unselected control stocks (C-1 and C-3) that were derived from this base population. Studies were done on crickets of the third-fifth laboratory generations. Frequencies of the LW morph in females of these stocks during these generations ranged from 0.60 to 0.69 in $C-1(N=120-148$ each generation) and from 0.49 to 0.75 in $\mathrm{C}-3$ (N=95-212 each generation).

Crickets were reared approximately $40-60$ per 10 gallon aquarium at $30^{\circ} \mathrm{C}$ under a $16 \mathrm{~L}: 8 \mathrm{D}$ photoperiod until late in the last instar after which they were subsequently held at $28^{\circ} \mathrm{C}$ under the same photoperiod. Approximately 100-150 males and 100-150 females were used as breeders each generation. All individuals were fed a standard wet diet (Mole and Zera, 1994). Crickets used for experiments were checked for molting at 24-h intervals and equal numbers of newly molted males and females were housed together at a density of 6 per 1-gallon box or 12 per 3-gallon box. No oviposition material was provided and hence females retained their eggs.

\subsection{Sampling schedule and hemolymph collection and processing}

Juvenile hormone titers were measured in females that were 3,5 , and 7 days old (molt to adult=day 0 ). These days were chosen because they span the period of development during which ovaries first begin to grow to a time when ovarian mass is substantially greater in flightless females [either SW or LW $(\mathrm{H})$ ]. All hemolymph samples were collected within the first $10 \mathrm{~h}$ of the 16$\mathrm{h}$ light cycle. 12.5-25 $\mu \mathrm{l}$ hemolymph from individual females were collected in micropipettes from cuts in the cerci and legs of G. firmus. Hemolymph was blown into a test tube containing $300 \mu 190 \%$ methanol in water. Samples were sonicated for 3-5 s and JH was extracted twice with $600 \mu \mathrm{l}$ hexane. Hexane extracts were stored in glass vials at $-80^{\circ} \mathrm{C}$ until radioimmunassay (RIA). Bled females were stored at $-80^{\circ} \mathrm{C}$ until dissection, when reproductive characteristics and flight muscle phenotype were determined. Background experiments showed that these features did not change during the time crickets were frozen. JH titers were measured in 3-20 individual females of each morph from each line on each day.

\subsection{Juvenile hormone RIA: assay conditions}

Juvenile hormone titers were determined using a RIA specific for the naturally occurring (10R) enantiomer of juvenile hormone (Hunnicutt et al., 1989; Huang et al., 1994). JH III has been identified as the only juvenile hormone in G. firmus (Yadlowski, 1994) and other grillids (Loher et al., 1983; Koch and Hoffman, 1985; Zera et al., 1989). Prior to RIA, individual hemolymph sam- ples were first dried in a vacuum centrifuge and $50 \mu \mathrm{l}$ of methanol was added to each sample. A 4- $\mu$ l aliquot was transferred to another tube containing $200 \mu \mathrm{l}$ of premixed antiserum in gel-PBST $(0.1 \%$ Knox's gelatin in $0.05 \mathrm{M}$ phosphate buffer, $\mathrm{pH} 7.3 ; 0.9 \% \mathrm{NaCl} ; 0.01 \%$ Triton X-100) and $4000 \mathrm{dpm}$ of $\left[10^{-3} \mathrm{H}\right]-\mathrm{JH}$ III. The final dilution of anti-JH III rabbit serum was 1:16,000. Tubes were incubated for 8-12 h at room temperature. After cooling in an ice bath for $5 \mathrm{~min}$, unbound radiolabelled JH III was separated from bound JH III by adding 500 $\mu l$ dextran-coated charcoal, incubating for $5 \mathrm{~min}$, and centrifuging for $5 \mathrm{~min}$ at $1500 \mathrm{~g}$ at $4^{\circ} \mathrm{C}$. Radioactivity in the supernatant was due to radiolabeled JH III bound to antiserum while radioactivity in the pellet was due to charcoal adsorption of unbound radiolabeled hormone. The supernatant was transferred to scintillation vials and dpm (disintegrations per minute) quantified by liquid scintillation spectrometry (RackBeta 1219). Under the assay conditions described above, antiserum bound approximately $25 \%$ radiolabeled JH III (50\% 10-R JH III) in the absence of unlabelled ligand, which is optimal for this assay.

\subsection{Juvenile hormone RIA: validation}

A standard curve based on seven concentrations of racemic JH III ranging from 1.5 to $1500 \mathrm{pg}$ was constructed for each assay (see Figure 1 for representative curve). Parameters of the standard curve were estimated by nonlinear regression (Prism 2.0, GraphPad Inc.). Data points fit the standard curve very well (mean $r^{2}=0.9959 \pm .0003$, $N=8$ curves), enabling accurate estimates of the juvenile hormone titer in hemolymph samples of individual G. firmus. The concentration of racemic JH III standards was multiplied by 0.5 since they contain only $50 \%$ of the $10 \mathrm{R}$ enantiomer that is recognized by the anti-JH antibody (Hunnicutt et al., 1989).

To determine whether RIA of G. firmus hemolymph extracts is affected by hemolymph lipids, 28 individual hemolymph extracts were divided in half and one half of each sample was passed through a Prep-Sep column to remove lipids. The extract to be passed through the column was dried in a vacuum centrifuge and resuspended in $1 \mathrm{ml} \mathrm{100 \%}$ methylene chloride. The silica gel Prep-Sep column was washed with $2 \mathrm{ml}$ methanol followed by $2 \mathrm{ml}$ methylene chloride. JH extracts were passed through the column and the column was washed with $1 \mathrm{ml}$ methylene chloride. The column was then washed with $2 \mathrm{ml} \mathrm{10 \%} \mathrm{methylene} \mathrm{chloride} \mathrm{in} \mathrm{methanol}$ which elutes JH but retains lipids. Radiolabeled JH III (5000 dpm) was added to three samples to monitor JH recovery during Prep-Sep purification. More than 97\% of JH III was recovered in the $10 \%$ methylene chloride wash. JH titers estimated in the purified and unpurified sample halves were very similar $(r=0.90, P<0.0001$; see 
Figure 1). This strong correlation indicates that determinations of the hemolymph JH titer of G. firmus using this RIA are only minimally affected by the presence of hemolymph lipids. A similar situation as has been demonstrated for honey bees (Huang et al., 1994) and burying beetles (Trumbo et al., 1995).

To check the specificity of the RIA, four separate extracts of hemolymph from adult female G. firmus and two JH III standards (5 ng) were individually fractionated by normal phase HPLC as described in Trumbo et al. (1995). Prior to HPLC, a known amount of radiolabeled JH III was added to the sample. A portion of each sample was saved and the remainder was injected into the column and seven fractions that bracketed the
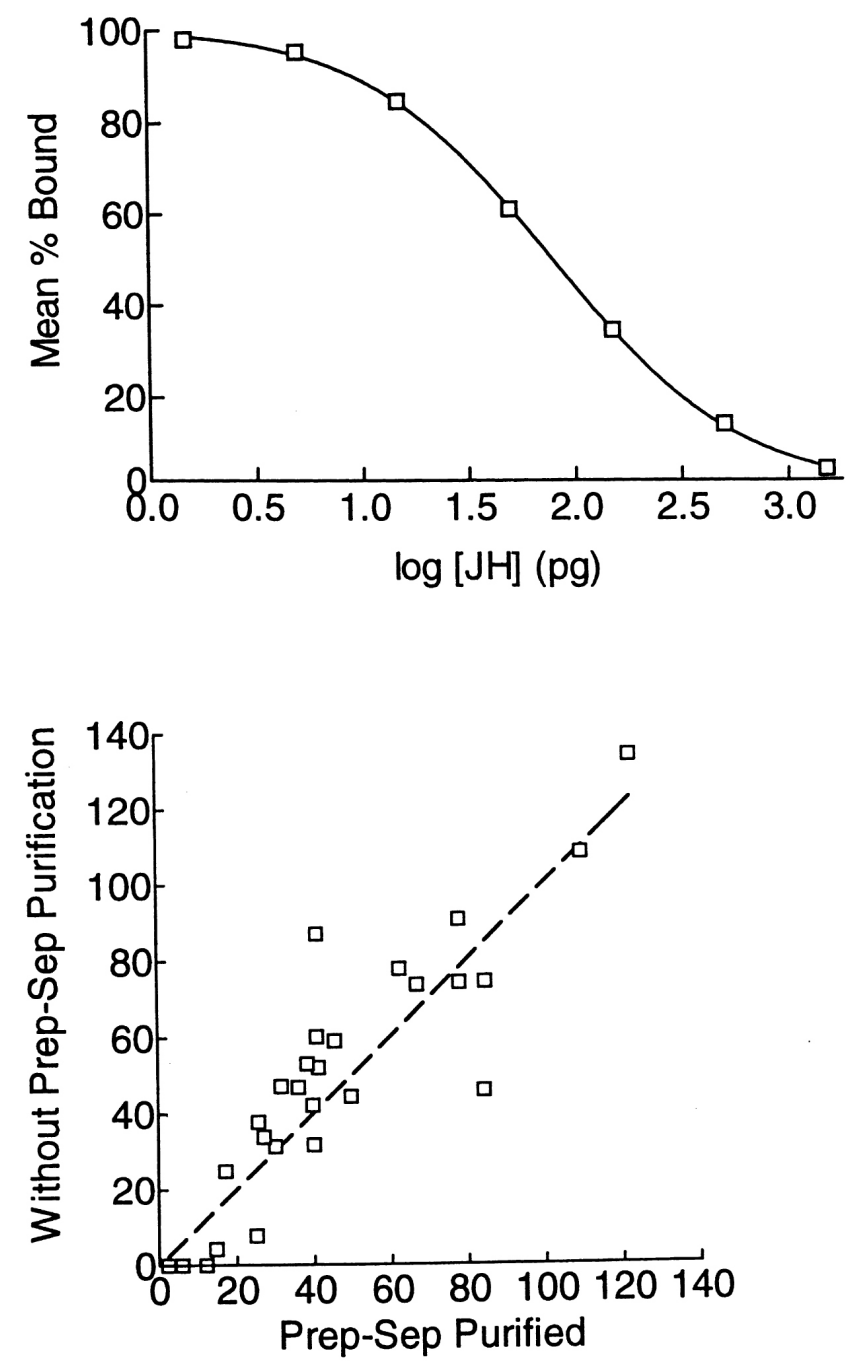

Figure 1. Top panel: typical standard curve for RIA of racemic juvenile hormone-III. Values are means $( \pm S E M)$ of three replicates. SEMs are smaller than the symbols. See Section 2 for details. Bottom panel: effect of Prep-Sep purification on the estimation of juvenile hormone in extracts of hemolymph from G. firmus. Symbols represent individual hemolymph extracts that were divided in half and subjected to RIA with or without passage through a Prep-Sep column. Units on the Xand $Y$-axes are picograms of juvenile hormone. The dotted line indicates values that are equal for purified and unpurified portions of hemolymph extracts. elution of JH III from the column were collected. Aliquots of the unfractionated portion and each HPLC fraction were individually counted to determine the elution time of $\mathrm{JH}$ and its recovery. Other aliquots of these samples were analyzed by RIA to determine their JH content. The amount of ${ }^{3} \mathrm{H}-\mathrm{JH}$ III (added to monitor recovery) present in each sample was taken into account when calculating the amount of $\mathrm{JH}$. These data were then used to calculate the percentage of the immunoreactive material in the unfractionated sample that co-migrated with JH III, after correcting for the recovery of JH from HPLC. In all of the samples $(n=4)$, immunoreactive material was only detected in the two HPLC fractions that also contained ${ }^{3} \mathrm{H}-\mathrm{JH}$ III. The immunoreactive material in these fractions accounted for all $(102.0 \pm 17.4 \%)$ of the activity observed in the unfractionated sample. The co-migration of all immunoreactivity with JH III in normal phase HPLC is similar to results obtained by Huang et al. (1994) and Trumbo et al. (1995).

\subsection{Dissections}

Ovarian mass, number of postvitellogenic eggs, longest terminal oocyte length (average of five terminal oocytes in each individual) and flight muscle phenotype (pink, fully developed vs. white, reduced) were determined for individuals whose JH titers were determined. Muscle color is strongly correlated with muscle size and can be used as an indicator of muscle histolysis in LW females (see Zera et al., 1997).

\subsection{Statistical analyses}

Variation in the $\mathrm{JH}$ titer between the three morphs (MORPH), three days of adulthood (DAY) and the two control stocks (BLOCK) was determined by Analysis of Variance (ANOVA; Sokal and Rohlf, 1981). BLOCK was a random effect and DAY and MORPH were fixed effects. Distributions of JH titers were significantly skewed and titers were normalized prior to analysis by transforming them to $\log [\mathrm{JH}$ titer+1] $(\mathrm{pg} / \mu \mathrm{l})$. One was added to the estimated $\mathrm{JH}$ titer in order to circumvent the technical problem of log transforming 0 values (see Sokal and Rohlf, 1981). The initial ANOVA showed no significant effect of BLOCK or any two- or three-way interactions involving BLOCK. Thus, BLOCK was removed from the model (i.e. data from the two control lines were pooled) and data were analyzed by two-way ANOVA. In addition, untransformed $\mathrm{JH}$ titer data were analyzed by the non-parametric Mann-Whitney U-test. The overall comparison of JH titers among the three morphs was followed by two-way comparisons of titers between the various morphs $[\mathrm{LW}(\mathrm{F})$ vs. SW, $\operatorname{LW}(\mathrm{F})$ vs. $\operatorname{LW}(\mathrm{H})$, and $\mathrm{LW}(\mathrm{H})$ vs. SW]. Since these comparisons were planned contrasts, probabilities were not adjusted for multiple comparisons. 
Associations between reproductive traits (which could not be normalized by various transformations) and the $\mathrm{JH}$ titer were analyzed using the nonparametric Spearman rank correlation (Sokal and Rohlf, 1981).

The various reproductive traits (e.g. \% ovarian mass), in addition to not being normally distributed, also differed between the two control stocks. Because of these complications, reproductive data were analyzed by Mann-Whitney U-tests in which traits were compared between pairs of morphs of each of the two control lines separately for each day of adulthood. As was the case for the JH titers, analyses of reproductive characteristics were planned contrasts and were not corrected for multiple comparisons. Finally, comparisons between LW(F) and SW or $\mathrm{LW}(\mathrm{H})$ were one-tailed with the a priori expectation that reproductive traits or $\mathrm{JH}$ titers would be higher in $\operatorname{LW}(\mathrm{H})$ or SW. This expectation is based on many studies in other insects, as well as a previous study in G. firmus, which have documented enhanced reproduction in the morph with reduced flight muscles compared with the flight-capable morph (Zera et al., 1997; Zera and Denno, 1997). By contrast, we had no expectation concerning the directionality of differences for various reproductive traits or the $\mathrm{JH}$ titer between $\mathrm{LW}(\mathrm{H})$ and SW morphs. Hence these comparisons were two-tailed.

\section{Results}

\subsection{Reproductive characteristics of wing and flight-muscle morphs}

Ovarian masses (mean \pm SEM $\%$ body wet mass) as a function of age for the three morphs of G. firmus from the two unselected (control) stocks are presented in Figure 2. Statistics for longest terminal oocyte length and number of postvitellogenic eggs are reported in Table 1. Ovarian mass and number of postvitellogenic eggs consistently increased over days 3, 5, and 7 for all three morphs. Two distinct profiles were evident for these reproductive traits. One profile of enhanced reproduction was found in SW and $\mathrm{LW}(\mathrm{H})$ morphs. This profile differed markedly from that of delayed reproduction in LW(F) females. On day 3 , ovarian mass was significantly higher in females with reduced flight muscles [LW(H) or SW] compared with females with pink flight muscles [LW(F)] for both C-1 and C-3 lines $(P<0.018$ for three of four Mann-Whitney U-tests). The one exception was day 3, C-3 crickets in which ovarian mass did not differ between LW females with white $[\mathrm{LW}(\mathrm{H})]$ and pink [LW(F)] flight muscles $(P>0.10$; one-tailed Mann-Whitney U-test). Postvitellogenic eggs were not observed in any morph on day 3. On days 5 and 7, all females with reduced flight muscles [SW or $\mathrm{LW}(\mathrm{H})$ ] had significantly larger ovaries and held a significantly greater number of postvitellogenic eggs in their ovaries than individuals with functional flight muscles $[\mathrm{LW}(\mathrm{F})](P<0.01$ for each one-tailed Mann-Whitney U-test). The two morphs with white flight muscles [SW and $\mathrm{LW}(\mathrm{H})$ ] did not differ in ovarian mass or number of postvitellogenic eggs on any day measured $(P>0.20$ for each two-tailed, Mann-Whitney U-test).

Patterns of terminal oocyte growth differed between the two control lines (Table 1). C-1 morphs with reduced flight muscles $[\mathrm{LW}(\mathrm{H})$ and $\mathrm{SW}]$ had significantly longer terminal oocytes than $\mathrm{LW}(\mathrm{F})$ females on days 3 and 5 $(P<0.02$ for each one-tailed Mann-Whitney U-test), but not on day 7 ( $P>0.40$ for each one-tailed Mann-Whitney U-test). In contrast, morphs of C-3 did not differ significantly in oocyte length on any day $(P>0.10$ for each one-tailed Mann-Whitney U-test). Examination of the data indicates that, in general, terminal oocytes approached maximum length by day 5 for females with reduced flight muscles $[\mathrm{LW}(\mathrm{H})$ and $\mathrm{SW}]$ and that terminal oocyte length in $L W(F)$ females on day 7 was slightly less than or equal to that of day $5 \mathrm{LW}(\mathrm{H})$ or $\mathrm{SW}$ females.

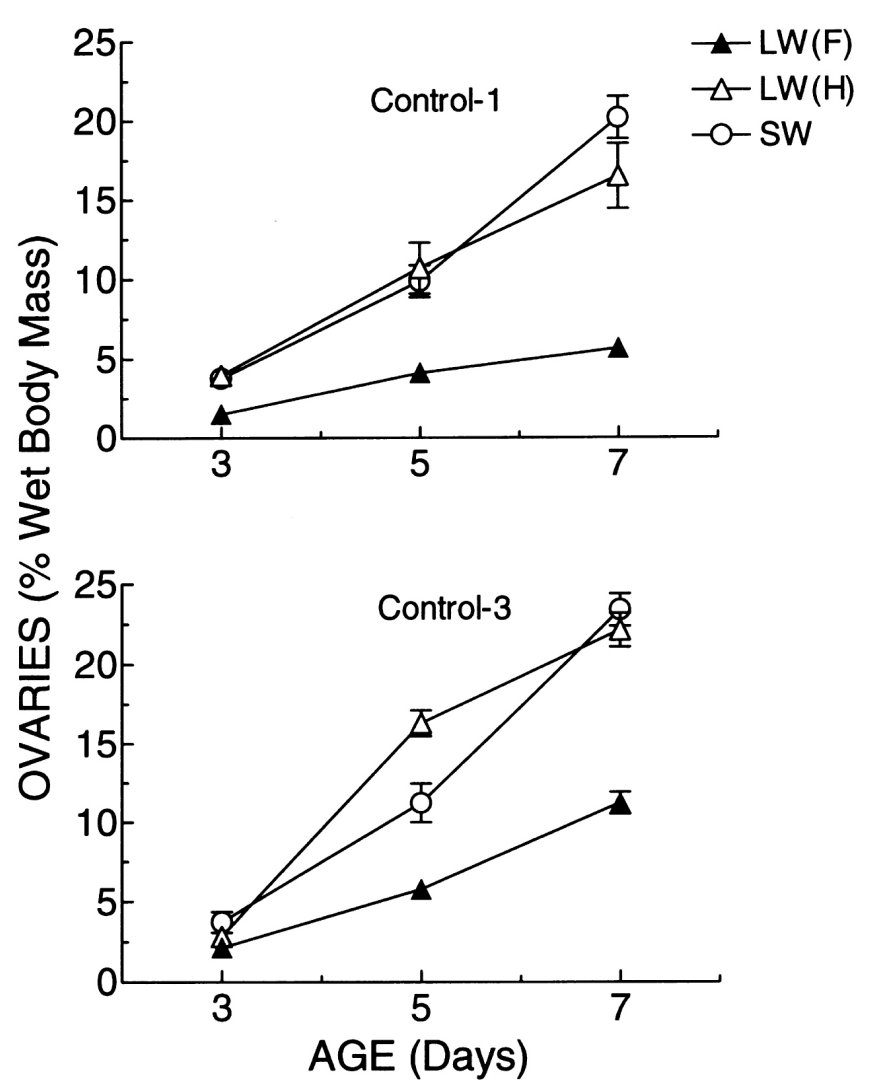

Figure 2. Ovarian mass (as \% body wet mass) for wing and flight muscle morphs of G. firmus from two control lines as a function of adult age. Day $0=$ molt to adult. $L W(F)=$ long-winged with functional flight muscles, $\mathrm{LW}(\mathrm{H})=$ long winged with histolyzed flight muscles, SW=short-winged with underdeveloped flight muscles. See Section 2 for line designations. For each control line on each day of adulthood, sample size ranges were 5-20 (median=14), 3-14 (median=5) and 1020 (median=15) for LW(F), LW(H), and SW females, respectively. 
Table 1. Terminal oocyte length and number of postvitellogenic eggs in wing and flight muscle morphs of the two control stocks of G. firmus

\begin{tabular}{|c|c|c|c|c|c|c|}
\hline & \multicolumn{3}{|c|}{ Control-1 } & \multicolumn{3}{|l|}{ Control-3 } \\
\hline & $\mathrm{LW}(\mathrm{F})^{\mathrm{a}}$ & $\mathrm{LW}(\mathrm{H})$ & SW & $\mathrm{LW}(\mathrm{F})$ & $\mathrm{LW}(\mathrm{H})$ & SW \\
\hline \multicolumn{7}{|c|}{ Longest terminal oocyte length $(\mathrm{mm})$} \\
\hline Day-5 & $\begin{array}{l}2.3 \pm 0.2 \\
(2.2,16)\end{array}$ & $\begin{array}{l}3.3 \pm 0.2 \\
(3.4,4)\end{array}$ & $\begin{array}{l}3.1 \pm 0.7 \\
(3.3,16)\end{array}$ & $\begin{array}{l}3.2 \pm 0.2 \\
(3.3,10)\end{array}$ & $\begin{array}{l}3.6 \pm 0.0 \\
(3.6,5)\end{array}$ & $\begin{array}{l}3.3 \pm 0.0 \\
(3.3,10)\end{array}$ \\
\hline \multicolumn{7}{|c|}{ Number of post-vitellogenic eggs } \\
\hline Day-5 & $\begin{array}{l}33 \pm 8 \\
(23,9)\end{array}$ & $\begin{array}{l}77 \pm 13 \\
(74,3)\end{array}$ & $\begin{array}{l}56 \pm 5 \\
(52,15)\end{array}$ & $\begin{array}{l}22 \pm 8 \\
(18,10)\end{array}$ & $\begin{array}{l}110 \pm 17 \\
(114,5)\end{array}$ & $\begin{array}{c}84 \pm 19 \\
(74,10)\end{array}$ \\
\hline
\end{tabular}

$\mathrm{a} L W(\mathrm{~F})=$ long-winged with functional flight muscles, $\mathrm{LW}(\mathrm{H})=$ long-winged with histolyzed flight muscles, $\mathrm{SW}=$ short-winged with underdeveloped flight muscles.

$\mathrm{b}$ Grand mean $\pm(\mathrm{SEM})$ of the mean of five longest terminal oocytes per cricket.

c Numbers in parentheses are the median followed by sample size (number of individual crickets).

\subsection{Juvenile hormone titers}

Median $\mathrm{JH}$ titers and mean $\log [\mathrm{JH}+1]$ titers as a function of age for the three morphs of G. firmus are presented in Figure 3. Titers did not differ significantly between the two control lines and hence titer data from these lines were combined for presentation and further analysis (see Section 2). Median JH titers ranged from 2 to $13 \mathrm{pg} / \mathrm{\mu l}$ in the morphs across the 3 days measured. Except for two cases discussed below, statistical analyses of the log-transformed JH titers by ANOVA or the untransformed titers by the non-parametric Mann-Whitney U-test gave identical results. The three morphs differed significantly in their JH titers (Table 2). LW individuals with pink flight muscles [LW(F)] had the lowest $\mathrm{JH}$ titers and SW individuals had the highest JH titers. LW individuals with white flight muscles [LW(H)] had intermediate $\mathrm{JH}$ titers that were closer to the SW than the $\mathrm{LW}(\mathrm{F})$ morph on most days (Figure 3). When tested separately, the $\mathrm{SW}$ morph had a significantly higher JH titer than the LW morph with pink flight muscles across all days and on days 5 and 7, but not on day 3 (Table 2). Titer differences between the LW morphs with white and pink flight muscles $[\mathrm{LW}(\mathrm{H})$ vs. $\mathrm{LW}(\mathrm{F})]$ were nonsignificant across all days when tested by ANOVA (Table 2) but differed significantly when tested by a one-tailed Mann-Whitney U-test $\left(U=1096, n_{1}=81, n_{2}=35, P<0.03\right)$. Titers did not differ between these two morphs on any single day when tested by ANOVA (Table 2) and only differed marginally on day 5 when tested by a one-tailed
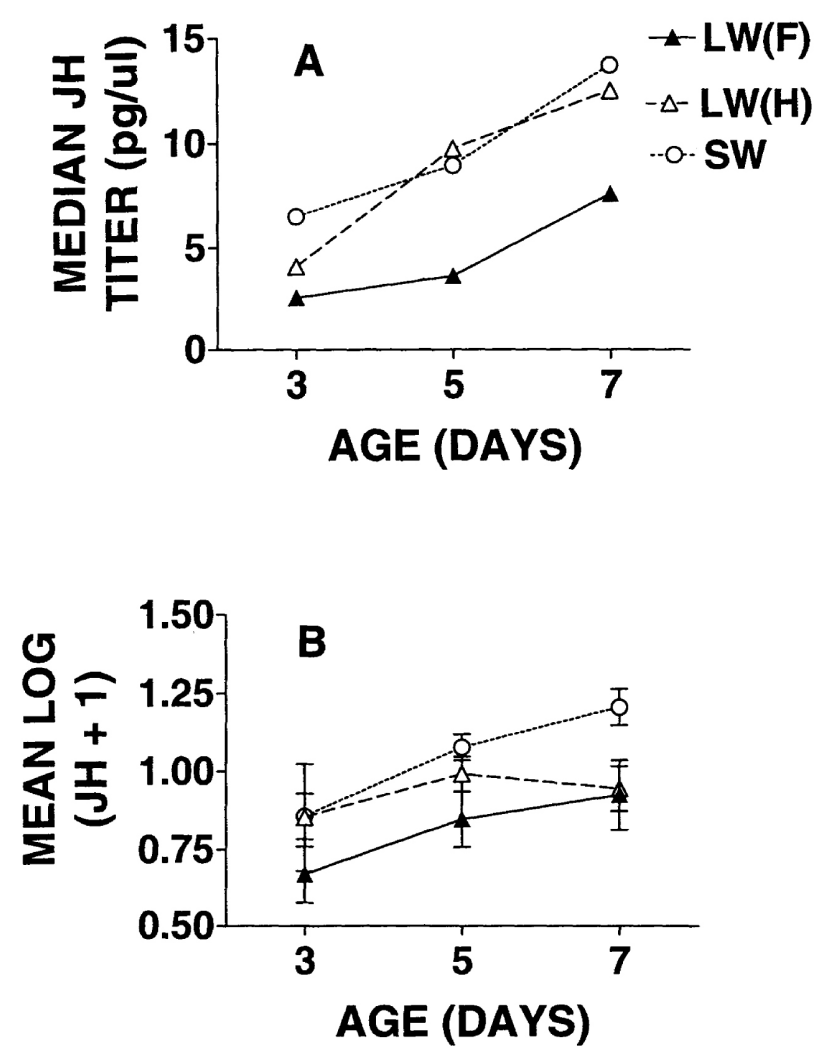

Figure 3. Median (panel A) and mean $( \pm$ SEM) (panel B) hemolymph $\mathrm{JH}$ titer in wing and flight muscle morphs of G. firmus. Symbol designations are the same as those in Figure 2. Since JH titers did not differ significantly between the two control lines, pooled data for both lines are presented. Sample sizes on each day were 25-28 [LW(F)], 6$21[\mathrm{LW}(\mathrm{H})]$, and $30(\mathrm{SW})$. Note that $\mathrm{JH}$ titers in panel B were transformed to $\log (\mathrm{JH}+1)$ values (see Section 2). 
Table 2. Analysis of JH titer variation among morphs of G. firmus measured on different days of adulthood

\begin{tabular}{|c|c|c|c|}
\hline Morphs compared & \multicolumn{3}{|c|}{ Source of variation } \\
\hline All $[\mathrm{LW}(\mathrm{F}), \mathrm{LW}(\mathrm{H}), \mathrm{SW}]^{\mathrm{a}}$ & $7.1(2,197) b^{* * *}$ & $4.0(2,197)^{\star *}$ & \\
\hline LW(F) vs. SW & $13.1(1,165)^{* * *}$ & $7.5(2,75)^{\star * *}$ & \\
\hline LW(F) vs. LW(H) & $1.3(1,110), \mathrm{ns}$ & $1.0(2,110), \mathrm{ns}$ & \\
\hline \multicolumn{4}{|c|}{ B. Variation between morphs tested on individual days } \\
\hline & Day 3 & Day 5 & Day 7 \\
\hline LW(F) vs. SW & $2.6(1,56), \mathrm{ns}$ & $6.3(1,53)^{* *}$ & $5.2(1,56)^{* *}$ \\
\hline LW(F) vs. LW(H) & $0.8(1,32), \mathrm{ns}$ & $0.8(1,31), \mathrm{ns}$ & $0.0(1,47) \mathrm{ns}$ \\
\hline LW $(\mathrm{H})$ vs. SW & $0.0(1,34), \mathrm{ns}$ & $1.0(1,36), \mathrm{ns}$ & $8.1(1,49)^{* *}$ \\
\hline
\end{tabular}

a Morph designations: $\mathrm{LW}(\mathrm{F})=$ long-winged with functional flight muscles; $\mathrm{LW}(\mathrm{H})=$ long-winged with histolyzed flight muscles; $\mathrm{SW}=$ shortwings with underdeveloped flight muscles (see Section 2).

b Values outside of parentheses are F-ratios from ANOVA of (log+1)-transformed titers while values inside parentheses are degrees of freedom. Comparisons of all morphs and LW(H) vs. SW were two-tailed while comparisons between LW(F) and SW or LW $(\mathrm{H})$ were one-tailed (see Section 2). No significant morph $\times$ day interactions were observed.

*** $P<0.005 ;{ }^{* *} P<0.025 ;{ }^{*} P<0.05 ;$ ns $=$ non-significant $(P>0.1)$

Mann-Whitney U-test $\left(U=134, n_{1}=33, n_{2}=12, P=0.05\right)$. Titers did not differ between the two morphs with white flight muscles $[\mathrm{LW}(\mathrm{H})$ and $\mathrm{SW}]$ across all days, or on any individual day with one exception. On day 7, the SW morph had a significantly higher titer than the LW(H) morph (Table 2).

\subsection{Correlations between the juvenile hormone titer and re- productive characters}

Scatterplots of JH titers and reproductive traits [ovarian mass (as \% body mass), terminal oocyte length, and number of postvitellogenic eggs] are presented in Figure 4 and Figure 5. Data points in Figure 4 are measurements in individuals while Figure 5 represents the mean values for each of the three morphs calculated for each of the two stocks on each of the 3 days of adulthood ( $N=$ 18 means). The JH titer was significantly correlated with ovarian mass and terminal oocyte length for all individuals $(r=0.31, P<0.001$ and $r=0.26, P=0.001$, respectively; $N=206$ ) but was not significantly correlated with the number of postvitellogenic eggs $(r=0.13, P=0.22 ; N$ $=206$ ) (Figure 4). These correlations were essentially the same when $\operatorname{LW}(\mathrm{F})$ females were tested with only one of the types of flightless morph. For example, the correlation between JH titer and ovarian mass for the sample without $\mathrm{LW}(\mathrm{H})$ females was $r=0.38, P<0.001(N=$ 171), while the correlation for the sample without SW females was $r=0.26, P<0.005(N=116)$. Correlations were substantially higher between mean $\mathrm{JH}$ titers and mean values for the various reproductive traits (values averaged over individuals of a particular morph of particu- lar stock for a particular day of adulthood) $[r=0.62, P$ $=0.004$ for ovarian mass, $r=0.58, P=0.011$ for terminal oocyte length, and $r=0.57, P=0.014$ for number of postvitellogenic eggs (Figure 5)].

\section{Discussion}

\subsection{General aspects}

4.1.1. JH titer in G. firmus compared with other insects Median JH titers in G. firmus females derived from unselected control lines during the first week of adulthood (3-14 pg/ul; Figure 3) are at the lower end of the range of titers reported for females of the cricket species Teleogryllus commodus $(8-60 \mathrm{pg} / \mathrm{ul})$ and Acheta domesticus (20-70 pg/ul) and the locust, Locusta migratoria (4-30 pg/ul) (Loher et al., 1983, 1987; Renucci and Strambi, 1983; Dale and Tobe, 1986). As mentioned previously (see Section 2), JH III is the only juvenile hormone found in G. firmus. JH titers reported in the present study and in studies mentioned above have been quantified by a variety of methods all of which appear to accurately measure JH. The RIA used in the present study (Hunnicutt et al., 1989) has been used successfully to quantify $\mathrm{JH}$ levels in unpurified hemolymph from bees (Huang et al., 1994) and a beetle (Trumbo et al., 1995). Furthermore, the Hunnicutt RIA has been validated against two other JH-RIAs (Goodman et al., 1990; Strambi et al., 1981) which, in turn, have been validated against the gas-chromatographic/ mass spectrophotometric (GC/MS) method [see Methods of Huang et al. (1994) for details]. The Strambi 

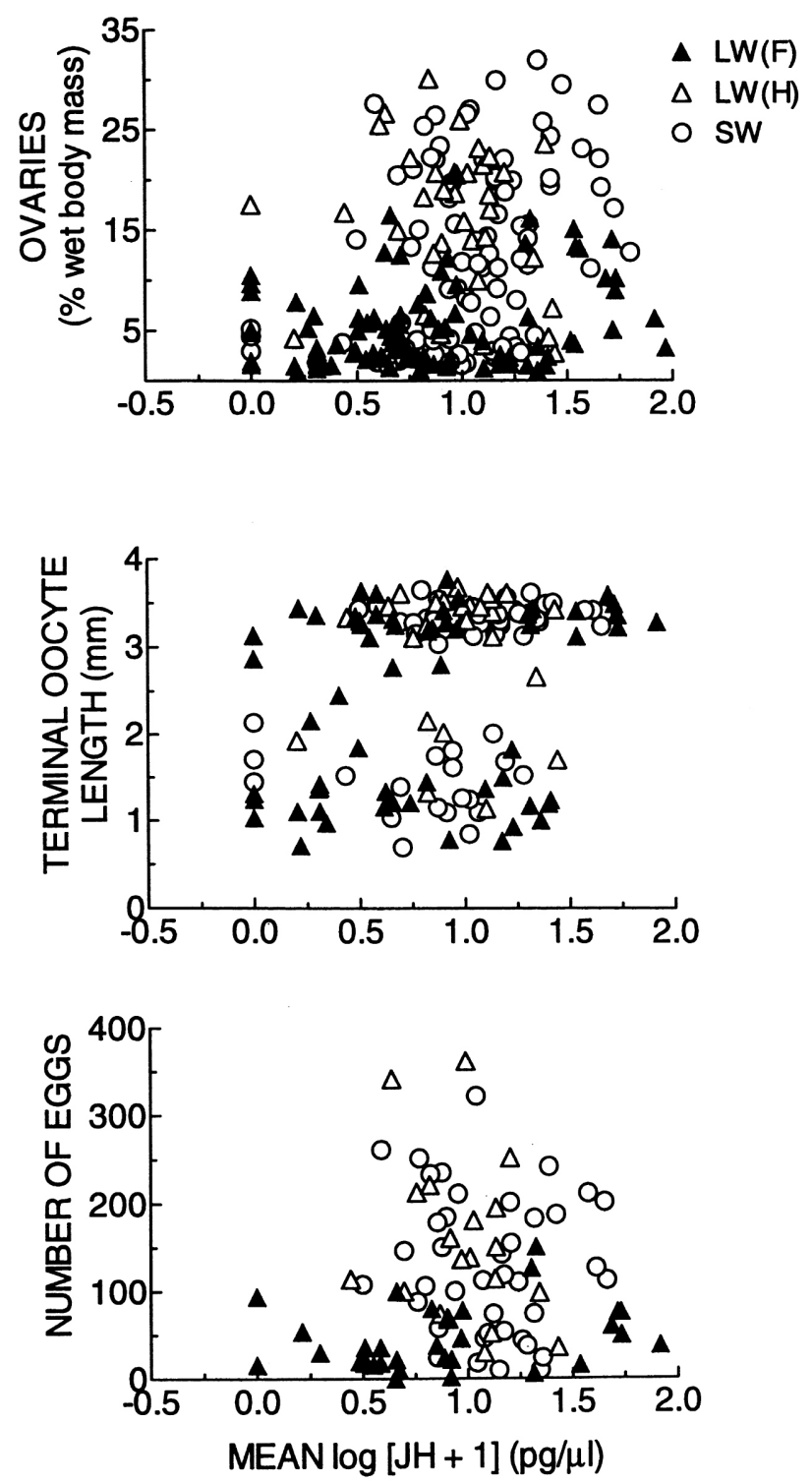

Figure 4. Scatterplots illustrating the relationship between ovarian mass (top panel), mean terminal oocyte length (middle panel), and number of postvitellogenic eggs (bottom panel) vs. JH titer in wing and flight muscle morphs of G. firmus. Symbol designations are the same as those in Figure 2 and refer to values for individual crickets.

RIA was used to quantify the JH titer in Acheta (Renucci and Strambi, 1983), while the GC/MS method was used to quantify JH in T. commodus (Loher et al., 1983, 1987) and L. migratoria (Dale and Tobe, 1986). The similar JH titers in G. firmus, Teleogryllus, Acheta and Locusta, combined with the results of our background RIA studies of HPLC-fractionated and Prep-Sep-purified hemolymph from G. firmus (see Section 2), indicate that the RIA used in the present study accurately measures $\mathrm{JH}$ in unpurified hemolymph from individual G. firmus.
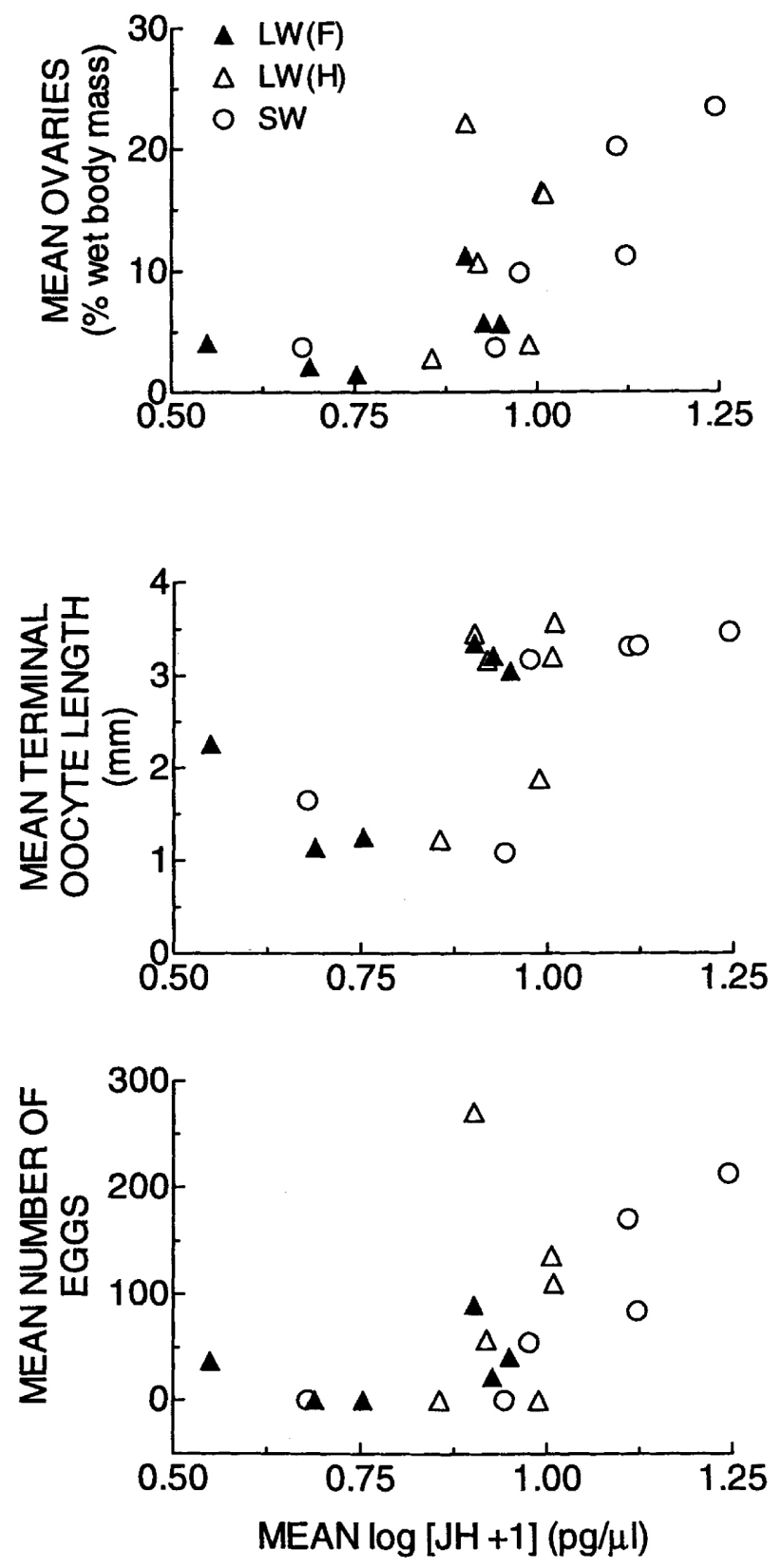

Figure 5. Scatterplots illustrating the relationship between mean ovarian mass (top panel), grand mean terminal oocyte length (middle panel) and mean number of postvitellogenic eggs (bottom panel) and hemolymph JH titer in wing and flight muscle morphs of G. firmus. Symbol designations are the same as those in Figure 2. Values represent means of crickets of a particular morph, day of adulthood, and line (control-1 or control-3) (see Section 2).

\subsection{JH titer and reproduction in G. firmus}

$\mathrm{JH}$ plays an important role in regulating oocyte growth and maturation in all crickets studied thus far (reviewed in Strambi et al., 1997). Prior to the present study, no information was available on the endocrine regulation of oocyte growth in G. firmus. Our results 
clearly implicate $\mathrm{JH}$ as a regulator of oocyte growth in this species. Ovarian mass, the number of postvitellogenic eggs, and JH titer all increased from day 3 to day 7. Furthermore, the $\mathrm{JH}$ titer was positively correlated with ovarian mass and length of terminal oocytes in individual G. firmus (see Section 3). We also have recently documented that topical application of either methoprene or JH III to the abdomen of intact LW G. firmus significantly increases ovarian mass (A. J. Zera, G. Cisper, and R. Koltz, unpublished data). On the other hand, the only moderate correlations between JH titer and various measures of reproduction in wing and flight muscle morphs indicate that $\mathrm{JH}$ is not the sole regulator of ovarian growth in this species. This is to be expected since numerous studies have identified hormones other than JH that influence ovarian growth (e.g. ecdysteroids and various neurohormones; Renucci and Strambi, 1981; Behrens and Hoffman, 1983; Nijhout, 1994; Strambi et al., 1997; Pener and Yerushalmi, 1998).

\subsection{Morph-specific reproduction and its endocrine regula-} tion: long-winged, flight capable $[L W(F)]$ vs. short-winged (SW) females

Elevated ovarian growth during early adulthood is one of the distinguishing features of the flightless morph of wing polymorphic insects (Harrison, 1980; Roff, 1984, 1986; Zera and Rankin, 1989; Zera and Denno, 1997; Zera et al., 1997). This elevated egg production during early adulthood is a major contributor to the greater overall fecundity of flightless vs. flight-capable females in many wing-polymorphic species (Roff, 1984, 1986; Zera and Rankin, 1989; Zera and Denno, 1997). Results obtained in the present study clearly document that SW females have substantially elevated ovarian mass and a greater number of post-vitellogenic eggs, compared with longwinged, flight-capable $[\mathrm{LW}(\mathrm{F})]$ females during the first week of adulthood (Figure 2; Table 1). The elevated ovarian mass of flightless females did not simply result from an earlier onset of ovarian growth. Rather, the growth rate of ovaries was about twice as high in flightless compared with flight-capable females during the first week of adulthood (Figure 2). The existence of larger ovaries in SW females during this period of adulthood is similar to results of previous studies of morph-specific reproduction in G. firmus (Roff, 1984; Zera et al., 1997).

For many years, an elevated JH titer has been proposed as the proximate cause of the elevated ovarian growth in flightless females of wing polymorphic species (Southwood, 1961; Wigglesworth, 1961; Lees, 1966; Nijhout and Wheeler, 1982; Hardie and Lees, 1985; Nijhout, 1994; Zera, and Denno, 1997). Although many studies have investigated this topic, all previous studies have been indirect. That is, an elevated JH titer in the flightless morph has been inferred from the results of ex- perimental manipulations, such as application of exogenous $\mathrm{JH}$ or $\mathrm{JH}$ analogues, or implantation of corpora allata into LW females. These manipulations have typically caused ovarian mass in long-winged females to increase in size to that of unmanipulated, flightless females, suggesting that an elevated JH titer causes the enhanced ovarian growth in flightless, unmanipulated females (Tanaka, 1994; Iwanaga and Tojo, 1986). However, these results are also consistent with other explanations, such as the induction by exogenous $\mathrm{JH}$ of unidentified hormones which are the true regulators of enhanced ovarian growth. The many feedback loops that typify endocrine physiology (Nijhout, 1994) make this a likely possibility, thus severely limiting the inferences about endogenous hormone titer variation that can be drawn solely from experimental manipulations (Zera and Tiebel, 1988; Zera and Denno, 1997).

The present study is the first to directly quantify JH titers in flight capable and flightless adult morphs of a wing polymorphic insect species. Moreover, titers were measured separately in the two LW morphs that differ dramatically in ovarian growth: long-winged females with pink functional flight muscles [LW(F)] and long-winged females with histolyzed, non-functional flight muscles $[\mathrm{LW}(\mathrm{H})]$. Our results show that the JH titer is significantly higher in flightless, SW females compared with flight-capable $[\mathrm{LW}(\mathrm{F})]$ females during the first week of adulthood (Figure 3 and Table 2). These results are clearly consistent with the idea that the greater ovarian growth in SW females during early adulthood is due, at least in part, to an elevated JH titer [JH titers in $\mathrm{LW}(\mathrm{H})$ females will be discussed below]. The positive correlation between $\mathrm{JH}$ titer and ovarian mass and size of terminal oocytes in individual G. firmus (Section 3) is also consistent with this idea.

Because no JH titer data are available for other wing polymorphic species, the extent to which the elevated JH titer in flightless adult G. firmus (Figure 3) also occurs in flightless females of other wing polymorphic species is unknown. Indeed there are very few data on $\mathrm{JH}$ titer variation in functionally similar types of polymorphism. For example, in the only other report of JH titer variation in aphids, Hardie et al. (1985) found that the JH III titer was elevated two-fold in whole-body extracts of the adult aphid, Megura viciae, that produced vivipera (parthenogenic females) vs. those that produced oviparae (sexual females). This suggested that $\mathrm{JH}$ might be involved in the regulation of sexual/parthenogenic polymorphism in this aphid species. However, the JH III titers were very low $(<0.2 \mathrm{ng} / \mathrm{g})$ and it is unclear whether JH-III is the main juvenile hormone in this species (Hardie et al., 1985). Juvenile hormone has been discussed for over four decades in the context of the endocrine regulation of phase polymorphism in locusts (Nijhout and Wheeler, 1982; Pener, 1991; Pener and Yerushalmi, 1998). However, other than the early 
study of Joly et al. (1977), which was done before the development of reliable methods for quantifying $\mathrm{JH}$ titers (see above), only very limited data on $\mathrm{JH}$ titer variation between phases has been reported. For example, Dale and Tobe (1986) found a two-fold elevation in the JH III titer in solitary vs. gregarious female L. migratoria during the first week of adulthood. Solitary females also had significantly larger ovaries than gregarious females during this time. However, the study of Dale and Tobe (1986), which primarily focused on phase-dependent differences in JH biosynthesis, only contained four individual $\mathrm{JH}$ titer measurements.

Although our results are consistent with the longstanding JH-morph reproduction hypothesis, they also indicate that an elevated $\mathrm{JH}$ titer does not appear to be the only factor responsible for the larger ovaries found in flightless females. Variation in the mean JH titer does account for a large proportion $(35-40 \%)$ of the variation in mean ovarian mass between morphs from the two control lines on various days of adulthood (Section 3 and Figure 5). However, variation in the JH titer among individuals accounts for only $10-15 \%$ of the total variation in ovarian mass $\left(r^{2}=0.096-0.144 ; r=\right.$ Spearman correlation between $\mathrm{JH}$ titer and \% ovarian mass; Section 3 and Figure 4). There are several possible explanations for the low-moderate correlation between $\mathrm{JH}$ titer and ovarian mass. One likely possibility is that variation in the JH titer is only one of several factors that causes the difference in ovarian growth between the flight-capable and flightless morphs. As mentioned above, there are numerous endocrine factors such as ecdysteroids and various neuropeptides which influence ovarian growth (see references above). Thus, an assessment of the importance of $\mathrm{JH}$ in morph-specific ovarian growth must await studies of these other potential regulators of ovarian growth.

Very recently, we have found that the JH titer varies diurnally in G. firmus (A. J. Zera, G. Cisper, and D. Katz, unpublished data). Diurnal change in the JH titer was unknown when the present study was conducted, and, there is no published report of diurnal variation in the JH titer except for Walker and Denlinger (1980). The existence of diurnal $\mathrm{JH}$ titer variation complicates an assessment of the existence, magnitude and functional significance of $\mathrm{JH}$ titer differences between wing and flight muscle morphs of G. firmus. For example, if hemolymph samples were taken during a period of time during which $\mathrm{JH}$ titers were changing, then titer differences between morphs may have been masked to some extent and hence underestimated. If this were the case, correlations between the JH titer and reproductive traits might also have been underestimated. Furthermore, observed titer differences between the morphs (Figure 3) may only exist during the restricted period of time during which the titers were measured. The extent of these complications can only be determined by more compre- hensive studies of G. firmus in which JH titers are measured during short periods of time throughout the photophase and scotophase. Such studies are currently in progress. Hence, we emphasize that $\mathrm{JH}$ titer variation among morphs of G. firmus reported in the present study (Figure 3) may underestimate actual titer differences between the morphs. Furthermore, differences among the morphs in the JH titer (Figure 3) may not exist during all periods of the photophase and scotophase. We also emphasize that our studies have focused exclusively on the endocrine regulation of morph-specific reproduction during the first week of adulthood.

\subsection{LW morphs with fully-developed $[L W(F)]$ and histolyzed $[L W(H)]$ flight muscles}

Until very recently, ecological and physiological studies of wing polymorphism have involved comparisons between morphs that vary in wing length (Zera et al., 1997). However, as mentioned previously (see Section 1 ), in many insect species, including G. firmus, LW females are a composite group of individuals some of which retain their flight muscles [LW(F)] and others of which histolyze their flight muscles when ovarian growth begins $[\mathrm{LW}(\mathrm{H})]$. $L W(\mathrm{H})$ females are more similar to $\mathrm{SW}$ than to $L W(F)$ females in a variety of key characteristics including ovarian growth, respiration rates of flight muscles and activities of enzymes in flight muscles (Figure 2; Zera et al., 1997, 1999). Because of these marked differences, it is essential to obtain measurements separately for these two types of LW morphs. Indeed, Zera et al. (1997) recently documented that the degree of enhancement of egg production due to flightlessness can be underestimated by as much as 50\% when reproductive differences between flight-capable $\mathrm{LW}(\mathrm{F})$ and flightless $\mathrm{LW}(\mathrm{H})$ morphs are not distinguished. The existence of two types of flightless morphs in the same species, that are produced by different mechanisms (blockage of flight muscle growth vs. muscle histolysis) during different life cycle stages (juvenile vs. adult), provides the opportunity to compare physiological features of flightless phenotypes that are the products of different evolutionary pathways to flightlessness (this point is discussed in more detail in Zera et al., 1997).

As was found in a previous study (Zera et al., 1997), ovarian growth is essentially identical in $\operatorname{LW}(\mathrm{H})$ and SW females and is substantially higher in each of these morphs than in LW(F) females (Figure 2). This corroborates our previous argument that reduction in flight muscles is more strongly associated with enhanced reproduction than is reduction in wings (Zera et al., 1997). By contrast, differences in the JH titer were greater between $\mathrm{LW}(\mathrm{F})$ and SW females than between $\mathrm{LW}(\mathrm{F})$ and $\mathrm{LW}(\mathrm{H})$ females (Section 3 and Figure 3). This suggests that the enhanced reproduction in the two differ- 
ent flightless morphs may be caused, in part, by modification of different endocrine mechanisms that regulate ovarian growth.

\subsection{Conclusions and future prospects}

In summary, we have documented significant variation in the JH titer among morphs of G. firmus that is correlated with variation in several reproductive measures (Figures 3-5 and Table 2). These results suggest that the increased JH titer during the first week of adulthood, at least in part, causes the elevated ovarian growth of the $\mathrm{SW}$ morph. To more precisely identify the role of JH titer variation in morph-specific reproduction in G. firmus, we are currently quantifying variation in the JH titer, variation in the ecdysteroid titer, and variation in the activity of regulators of the JH titer (juvenile hormone esterase) between adult wing morphs at specific points of the photophase and scotophase. Our previous studies of the endocrine regulation of wing polymorphism have focused on the regulation of morph development by JH during the last stadium in G. rubens and G. firmus. Despite considerable study, the importance of JH in regulating morph development remains uncertain (reviewed in Zera and Denno, 1997; Zera, 1999; Zera and Huang, 1999). JH titers are 10-100 fold higher in adult compared with juvenile Gryllus (e.g. compare titers in Figure 3 of this study with those of Figure 8 of Zera et al., 1989). Because of the much higher JH titer in adults, hormone titers can be quantified in individuals, in contrast to laststadium nymphs where hemolymph from at least 20 crickets must be pooled for a single titer measurement. Furthermore, titers clearly differ between adult morphs (Figure 3), in contrast to the only subtle titer variation between last-stadium morphs (Zera et al., 1989; Zera and Denno, 1997; Zera, 1999). Thus, JH regulation of morphspecific reproduction will likely be a more-tractable experimental problem than $\mathrm{JH}$ regulation of morph-specific development.

\section{Acknowledgements}

This research was supported by NSF grant IBN-9507388 to A. J. Zera. G. Cisper gratefully acknowledges a grant from the Orthopterist's Society which also supported this study. This paper is derived from a thesis submitted to the Graduate School, University of Nebraska-Lincoln, by G. Cisper in partial fulfillment of a Masters Degree in Biological Sciences.

\section{References}

Behrens, W., and Hoffman, K. H., 1983. Effects of exogenous ecdysteroids on reproduction in crickets Gryllus bimaculatus. International Journal of Invertebrate Reproduction 6, pp. 149-159.

Dale, J. F., and Tobe, S. S., 1986. Biosynthesis and titre of juvenile hormone during the first gonadotrophic cycle in iso- lated and crowded Locusta migratoria females. Journal of Insect Physiology 32, pp. 763-769.

Denno, R. F., Olmstead, K. L., and McCloud, E. S., 1989. Reproductive cost of flight capability: a comparison of life history traits in wing dimorphic planthoppers. Ecological Entomology 14, pp. 31-44.

Dingle, H., 1996. . Migration: the Biology of Life on the Move Oxford University Press, Oxford.

Goodman, W. G., Coy, D. C., Baker, F. C., Xu, L., and Toong, Y. C., 1990. Development and application of a radioimmunoassay for the juvenile hormones. Insect Biochemistry and Molecular Biology 20, pp. 357-364.

Hardie, J., Baker, F. C., Jamieson, G. C., Lees, A. D., and Schooley, D. A., 1985. The identification of an aphid juvenile hormone, and its titre in relation to photoperiod. Physiological Entomology 10, pp. 297-302.

Hardie, J., and Lees, A. D., 1985. Endocrine control of polymorphism and polyphenism. In: Kerkut, G. A., and Gilbert, L. I. Editors, 1985. Comprehensive Insect Physiology, Biochemistry and Pharmacology vol. 8 Pergamon Press, New York, pp. 441-490.

Harrison, R. G., 1980. Dispersal polymorphisms in insects. Annual Review of Ecology and Systematics 11, pp. 95-118.

Huang, Z. -Y., Robinson, G. E., and Borst, D. W., 1994. Physiological correlates of division of labor independent of age in honey bee colonies. Journal of Comparative Physiology 174, pp. 731-739.

Hunnicutt, D., Toong, Y., and Borst, D. W., 1989. A chiral specific antiserum for juvenile hormone. American Zoologist 29, p. 48a.

Iwanaga, K., and Tojo, S., 1986. Effects of juvenile hormone and rearing density on wing dimorphism and oocyte development in the brown planthopper, Nilaparvata lugens. Journal of Insect Physiology 32, pp. 585-590.

Joly, L., Hoffman, J., and Joly, P., 1977. Contrôle humoral de la différentiation phasaire chez Locusta migratoria migratorioides (R. and F.) (Orthoptera). Acrida 6, pp. 33-42.

Koch, P. B., and Hoffmann, K. H., 1985. Juvenile hormone and reproduction in crickets, Gryllus bimaculatus De Geer: corpus allatum activity (in vitro) in females during adult life cycle. Physiological Entomology 10, pp. 173-182.

Lees, A. D., 1966. The control of polymorphism in aphids. Advances in Insect Physiology 3, pp. 207-277.

Loher, W., Ruzo, L., Baker, F. C., Miller, C. A., and Schooley, D. A., 1983. Identification of the juvenile hormone from the cricket, Teleogryllus commodus, and juvenile hormone titre changes. Journal of Insect Physiology 29, pp. 585-589.

Loher, W., Schooley, D. A., and Baker, F. C., 1987. Influence of ovaries on JH titer in Teleogryllus commodus. Insect Biochemistry 17, pp. 1099-1102.

Mittler, T. E., 1991. Juvenile hormone and aphid polymorphism. In: Gupta, A. P. Editor, , 1991. Morphogenetic Hormones of Arthropods vol. 3 Rutgers University Press, New Brunswick, pp. 453-474.

Mole, S., and Zera, A. J., 1994. Differential resource consumption obviates a potential flight-fecundity trade-off in the sand cricket (Gryllus firmus). Functional Ecology 8, pp. 573-580.

Nijhout, F. J., and Wheeler, D., 1982. Juvenile hormone and the physiological basis of insect polymorphism. Quarterly Review of Biology 57, pp. 109-133. 
Nijhout, H. F., 1994. . Insect Hormones Princeton University Press, Princeton.

Pener, M. P., 1991. Locust phase polymorphism and its endocrine relations. Advances in Insect Physiology 23, pp. 1-80.

Pener, M. P., and Yerushalmi, Y., 1998. The physiology of locust phase polymorphism: an update. Journal of Insect Physiology 44, pp. 365-377.

Renucci, M., and Strambi, A., 1981. Evolution des ecdystéroides ovariens et hémolymphatiques au cours de la maturation ovarienne chez Acheta domesticus L. Comptes Rendus de l'Academie des Sciences 293, pp. 825-830.

Renucci, M., and Strambi, C., 1983. Juvenile hormone levels, vitellogenin and ovarian development in Acheta domesticus. Experientia 39, pp. 618-620.

Roff, D. A., 1984. The cost of being able to fly: a study of wing polymorphism in two species of crickets. Oecologia 63, pp. 30-37.

Roff, D. A., 1986. The evolution of wing dimorphism in insects. Evolution 40, pp. 1009-1020.

Sokal, R. R., and Rohlf, F. J., 1981. . Biometry (2nd ed. ed.), Freeman and Co, San Francisco.

Southwood, T. R. E., 1961. A hormonal theory of the mechanism of wing polymorphism in Heteroptera. Proceedings of the Royal Entomological Society of London 36, pp. 63-66.

Strambi, A., Strambi, C., and Cayre, M., 1997. Hormonal control of reproduction and reproductive behavior in crickets. Archives of Insect Biochemistry and Physiology 35, pp. 393-404.

Strambi, C., Strambi, A., de Riggi, M., and Delagge, M. A., 1981. Radioimmunoassay of insect juvenile hormones and their diol derivatives. European Journal of Biochemistry 118, pp. 401-406.

Tanaka, S., 1994. Endocrine control of ovarian development and flight muscle histolysis in a wing dimorphic cricket, Modicogryllus confirmatus. Journal of Insect Physiology 40, pp. 483-490.

Trumbo, S. T., Borst, D. W., and Robinson, G. E., 1995. Rapid elevation of juvenile hormone titer during behavioral assessment of the breeding resource by the burying beetle, Nicrophorus orbicollis. Journal of Insect Physiology 41, pp. 535-543.

Veazy, J. N., Kay, C. A. R., Walker, T. J., and Whitcomb, W. H., 1976. Seasonal abundance, sex ratio, and macroptery of field crickets in northern Florida. Annals of the Entomological Society of America 69, pp. 374-380.

Walker, G. P., and Denlinger, D. L., 1980. Juvenile hormone and moulting hormone titres in diapause- and non-diapause destined flesh flies. Journal of Insect Physiology 26, pp. 661-664.
Wigglesworth, V. B., 1961. Insect polymorphism - a tentative synthesis. In: Kennedy, J. S. (ed.), 1961. Insect Polymorphism Royal Entomological Society, London, pp. 103-113.

Wyatt, G. R., and Davey, K. G., 1996. Cellular and molecular actions of juvenile hormone. II. Roles of juvenile hormone in adult insects. Advances in Insect Physiology 26, pp. 1-155.

Yadlowski, D. E., 1994. Juvenile hormone esterase and correlated responses to selection: the physiological basis of wing dimorphism in the sand cricket, Gryllus firmus (Orthoptera: Gryllidae). M. Sc. Thesis, Department of Biology, Concordia University, Montreal, Canada. .

Zera, A. J., 1984. Differences in survivorship, development rate and fecundity between the longwinged and wingless morphs of the waterstrider, Limnoporus canaliculatus. Evolution 38, pp. 1023-1032.

Zera, A. J., 1999. The endocrine genetics of wing polymorphism in Gryllus: critique of recent studies and state of the art. Evolution 53, pp. 972-976.

Zera, A. J., and Denno, R. F., 1997. Physiology and ecology of dispersal polymorphism in insects. Annual Review of Entomology 42, pp. 207-231.

Zera, A. J., and Huang, Y., 1999. Evolutionary endocrinology of juvenile hormone esterase: functional relationship with wing polymorphism in the cricket, Gryllus firmus. Evolution 53, pp. 837-847.

Zera, A. J., and Rankin, M. A., 1989. Wing dimorphism in Gryllus rubens: genetic basis of morph determination and fertility differences between morphs. Oecologia 80, pp. 249-255.

Zera, A. J., Sall, J., and Grudzinski, K., 1997. Flight-muscle polymorphism in the cricket Gryllus firmus: muscle characteristics and their influence on the evolution of flightlessness. Physiological Zoology 70, pp. 519-529.

Zera, A. J., Sall, J., and Otto, K., 1999. Biochemical aspects of flight and flightlessness in Gryllus: flight fuels, enzyme activities and electrophoretic profiles of flight muscles from flight-capable and flightless morphs. Journal of Insect Physiology 45, pp. 275-285.

Zera, A. J., Strambi, C., Tiebel, K. C., Strambi, A., and Rankin, M. A., 1989. Juvenile hormone and ecdysteroid titers during critical periods of wing morph determination in Gryllus rubens. Journal of Insect Physiology 35, pp. 501-511.

Zera, A. J., and Tiebel, K. T., 1988. Brachypterizing effect of group rearing, juvenile hormone-III, and methoprene on winglength development in the wing-dimorphic cricket, Gryllus rubens. Journal of Insect Physiology 34, pp. 489-498. 